\title{
Improved Arousal and Motor Function Using Zolpidem in a Patient With Space-Occupying Intracranial Lesions: A Case Report
}

\author{
Martin Nicholas Bomalaski, MD, Sean Robinson Smith, MD
}

\begin{abstract}
Patients with disorders of consciousness (DOC) have profound functional limitations with few treatment options for improving arousal and quality of life. Zolpidem is a nonbenzodiazepine hypnotic used to treat insomnia that has also been observed to paradoxically improve arousal in those with DOC, such as the vegetative or minimally conscious states. Little information exists on its use in patients with DOC who have intracranial space-occupying lesions. We present a case of a 24-year-old man in a minimally conscious state due to central nervous system lymphoma who was observed to have increased arousal and improved motor function after the administration of zolpidem.
\end{abstract}

Level of Evidence: $V$

\section{Introduction}

Zolpidem is a nonbenzodiazepine hypnotic that is approved by the Food and Drug Administration for the short-term treatment of insomnia, with activity as a selective agonist of the omega-1 GABAa receptor subtype [1]. In 2000, Clauss et al [2] published a report of a young man who had been in a vegetative state (VS) for 3 years after a motor vehicle accident who regained consciousness 15 minutes after receiving $10 \mathrm{mg}$ of zolpidem. He was able to respond appropriately to environmental stimuli and communicate verbally with his family. The effects lasted approximately 3 hours before he returned to his VS. The effects were, however, reproducible with repeated administrations, dramatically improving the patient's function and quality of life [2]. Those with disorders of consciousness (DOC) have profound functional limitations and generally are unable to participate in their own self-care, creating a high caregiver and health system burden. Treatment options to improve cognitive awareness and overall function remain limited to a small number of agents, including amantadine, apomorphine, and levodopa, with limited evidence for their efficacy [3].

To date, zolpidem's paradoxical effects in this population primarily have been reported in patients with brain injury secondary to trauma, nontraumatic anoxia, or stroke [4]. There is little information on its use in patients with DOC who have intracranial spaceoccupying lesions. We present a case of a young man with central nervous system (CNS) lymphoma in a minimally conscious state (MCS) who was seen to have increased arousal and improved motor function after the administration of zolpidem.

\section{Case Presentation}

A previously healthy 24-year-old man presented to his local hospital with several weeks of worsening frontal headache, impaired coordination, and behavioral changes. Magnetic resonance imaging of his brain revealed a left frontal lobe lesion involving the basal ganglia with periventricular enhancement, cross-over at the corpus callosum, rightward midline shift, and satellite lesions in the left frontal lobe and cerebellum. Biopsy results were consistent with primary CNS B-cell lymphoma. Further workup revealed him to be positive for HIV, and he was started on antiretroviral therapy. After 1 week, he had multiple episodes of generalized seizure activity and was intubated for airway protection. At that time, he was reported to have right-sided hemiparesis and decreased arousal. On day 10, he became febrile and began decerebrate posturing and subsequently was transferred to our tertiary care 
hospital for further management. Ultimately, a tracheostomy was performed because of hypoxemic respiratory failure related to pneumonia.

Once weaned off of sedation and mechanical ventilation, he was found to be in an MCS, inconsistently following simple commands on his left, and answering yes/no questions using nonverbal gestures. Right-sided hemiparesis remained, and both upper and lower extremity spasticity worsened (Modified Ashworth Scale score of 3), for which he was started on baclofen $10 \mathrm{mg} 3$ times a day. He received methotrexate and rituximab chemotherapy soon after admission for CNS lymphoma, but further treatments were held due to his medical instability. He also received radiation therapy that ended on hospital day 74. He also was started on amantadine $100 \mathrm{mg}$ twice a day; however, there was no significant improvement in his level of cognitive awareness or participation in self-care, which remained total assist across all domains of the Functional Independence Measure. Repeat magnetic resonance imaging of the brain showed progression of his CNS lymphoma with increase in size of the frontal lobe and cerebellar lesions. Because of his poor functional status and disease progression, additional oncologic treatments were held.

A rehabilitation consult was requested to provide spasticity management and recommendations for mobility and decreased arousal. As part of the consult, on hospital day 126 a trial of zolpidem was suggested to improve arousal, given that the patient remained in an MCS, inconsistently following simple commands, and answering yes/no questions using nonvocal gestures.

The trial was performed in the morning using $10 \mathrm{mg}$ zolpidem administered via feeding tube. The Disability Rating Scale (DRS), an outcome measure that was developed to track functional recovery in patients with traumatic brain injury (lower score represents greater function) [5], and the Glasgow Coma Scale (GCS), which assesses level of consciousness in brain injured patients (greater score represents greater function) [6], were administered premedication and 45 minutes after administration. The DRS was selected to track changes in global function, whereas the GCS would focus on level of consciousness. Forty-five minutes was chosen because previous reports have shown that zolpidem often takes effect on patients with DOC around 30-60 minutes [4].

At 45 minutes postadministration of zolpidem, the patient initially appeared more somnolent, but quickly awoke with tactile stimulation, and was noticeably more interactive than before receiving the medication. He displayed functional object use, such as demonstrating how to drink from a cup and use a pen. There was improvement in both GCS and DRS, primarily in the domains of motor function (Table 1). He also could follow simple commands, such as waving to or giving thumbs up. Verbal scores were not able to be assessed, because an uncapped tracheostomy impaired his ability to speak or otherwise volitionally generate audible sounds.
Table 1

Assessment scores for zolpidem trials days 1 and 2

\begin{tabular}{|c|c|c|c|c|c|c|}
\hline \multirow[b]{2}{*}{ Assessment } & \multicolumn{2}{|c|}{ Day 1} & \multicolumn{4}{|c|}{ Day 2} \\
\hline & 0 & $45 \min$ & 1 & $1 \mathrm{~h}$ & $2 \mathrm{~h}$ & $4 \mathrm{~h}$ \\
\hline \multicolumn{7}{|l|}{ GCS } \\
\hline Motor & 4 & 6 & 4 & 4 & 6 & 6 \\
\hline Verbal & 1 & 1 & 4 & 1 & 4 & 2 \\
\hline Eyes & 4 & 4 & 1 & 1 & 1 & 1 \\
\hline Total & 9 & 11 & 9 & 6 & 11 & 10 \\
\hline \multicolumn{7}{|l|}{ DRS } \\
\hline Eyes & 0 & 0 & 0 & 2 & 0 & 2 \\
\hline Communication & 4 & 4 & 4 & 4 & 4 & 4 \\
\hline Motor & 2 & 0 & 2 & 2 & 0 & 0 \\
\hline Feeding & 3 & 2 & 3 & 3 & 2 & 2 \\
\hline Toileting & 3 & 3 & 3 & 3 & 3 & 3 \\
\hline Grooming & 3 & 3 & 3 & 3 & 3 & 3 \\
\hline Function & 5 & 5 & 5 & 5 & 5 & 5 \\
\hline Employability & 3 & 3 & 3 & 3 & 3 & 5 \\
\hline Total & 23 & 20 & 23 & 25 & 20 & 24 \\
\hline \multicolumn{7}{|l|}{ CRS-R } \\
\hline Auditory & & & 4 & 1 & 4 & \\
\hline Visual & & & 5 & 1 & 5 & \\
\hline Motor & & & 2 & 2 & 5 & \\
\hline Oromotor & & & 1 & 1 & 2 & \\
\hline Communication & & & 0 & 0 & 1 & \\
\hline Arousal & & & 1 & 1 & 2 & \\
\hline Total & & & 13 & 6 & 19 & \\
\hline
\end{tabular}

GCS = Glasgow Coma Scale; DRS = Disability Rating Scale; CRS-R = Coma Recovery Scale- Revised.

Because of the improvements seen in the first trial, a more extensive second trial was conducted 2 days later, this time also including the Coma Recovery ScaleRevised (greater score represents greater function), in addition to the GCS and DRS. The Coma Recovery ScaleRevised is a standardized functional scale validated in the DOC population [7]. Again, $10 \mathrm{mg}$ was administered in the morning, and assessments were made before and at 1, 2, and 4 hours after administration. GCS and DRS scores before administration of zolpidem ("baseline") were unchanged from those of the first trial. One hour after administration, he had a decline in function, appearing somnolent, not opening his eyes to painful stimuli, and not following commands. At 2 hours there was significant improvement from baseline in regards to arousal, communication, and motor function. He could again demonstrate functional object use with a cup and ball. He could consistently answer simple yes/no questions using thumbs up/down, respectively. At 4 hours, he had returned to near baseline. This trend was consistent across all outcomes (Table 1). The patient was discharged to a skilled nursing facility the following night, and passed away a few weeks later before any follow-up evaluations.

\section{Discussion}

To date, there is little known about the effects of zolpidem on arousal in patients with DOC who have 
intracranial space-occupying lesions. To our knowledge, this is one of the first reports of its off-label use in this population. In 2014, Du et al [8] performed a study in which they obtained single-photon positron emission tomography and cerebral state monitoring before and 1 hour after administering zolpidem to 165 subjects in a VS. Of these subjects, 40 had brain injury due to a space-occupying mass, typically either a tumor or hematoma. As a group they showed significant improvement across all measures. Average age among this group was 38.5 years and duration since time of injury was 62 days. Additional information, such as cause and location of the space-occupying lesion, or percentage of subjects who responded to zolpidem were not provided.

The omega-1 GABAa receptors that zolpidem binds selectively to are found in large quantities in the output structures of the basal ganglia, namely the globus pallidus interna and substantia nigra pars reticularis. Zolpidem's paradoxical effects in increasing arousal in patients with DOC may lie its ability to inhibit these inhibitory structures, thus helping to restore a normative balance between these pathways [9].

In regards to adverse effects, this was limited to sedation, which was seen at the 1-hour assessment during trial 2. Despite zolpidem's sedative properties, its occurrence in this population is less common than one might expect, with only 2 of 83 subjects showing signs of sedation in 2014 study by Whyte et al [4]. Zolpidem has been shown to reach peak plasma levels around 0.75-2.6 hours after administration, with a halflife of around 2.4 hours [1]. Our observation of improved function at $\mathbf{4 5}$ minutes after administration during the first trial and increased sedation at 1 hour followed by improvement at 2 hours on the second trial was most likely due to the natural fluctuations in level of consciousness seen in patients with DOC. There are some data to suggest a bimodal response; however, no conclusions can be made based on these limited data. Whyte et al observed a similar biphasic response in a zolpidem responder who had received $10 \mathrm{mg}$. They initially hypothesized that there may be an optimal therapeutic window; however, subsequent trials using 5 $\mathrm{mg}$ did not support this hypothesis, and the changes were attributed to behavioral variability, which is common among patients with DOC [10]. Although we cannot rule out the possibility that the changes we observed in response to zolpidem were due entirely to natural fluctuations, this seems less likely, considering similar changes were seen in 2 separate trials. The timing of changes we observed also were consistent with previous reports [4].

As a single case report, it is uncertain how predictive our findings are for a similar response in other patients with DOC who have space-occupying lesions. Future investigations are required to determine the safety and efficacy of zolpidem at various dosages in treating those with DOC in general and with space-occupying lesions in particular. Correlation of clinical response with functional neuroimaging may also aid in predicting who will respond to this intervention. We do, however, recognize the logistical challenges in conducting large trials in such an uncommon disorder with a high level of heterogeneity in regards to underlying brain injury.

\section{References}

1. Salvà $\mathrm{P}$, Costa J. Clinical pharmacokinetics and pharmacodynamics of zolpidem. Clin Pharmacokinet 1995;29:142-153.

2. Clauss RP, Güldenpfennig WM, Nel HW, Sathekge MM, Venkannagari RR. Extraordinary arousal from semi-comatose state on zolpidem. A case report. S Afr Med J 2000;90:68-72.

3. Ciurleo R, Bramanti P, Calabrò RS. Pharmacotherapy for disorders of consciousness: Are 'awakening'drugs really a possibility? Drugs 2013;73:1849-1862.

4. Whyte J, Rajan R, Rosenbaum A, et al. Zolpidem and restoration of consciousness. Am J Phys Med Rehabil 2014;93:101-113.

5. Rappaport M, Hall KM, Hopkins K, et al. Disability rating scale for severe head trauma: Coma to community. Arch Phys Med Rehabil 1982;63:118-123.

6. Teasdale G, Jennett B. Assessment of coma and impaired consciousness: A practical scale. Lancet 1974;304:81-84.

7. Giacino JT, Kalmar K, Whyte J. The JFK Coma Recovery ScaleRevised: Measurement characteristics and diagnostic utility. Arch Phys Med Rehabil 2004;85:2020-2029.

8. Du B, Shan A, Zhang $Y$, et al. Zolpidem arouses patients in vegetative state after brain injury: Quantitative evaluation and indications. Am J Med Sci 2014;347:178-182.

9. Schiff ND. Recovery of consciousness after brain injury: A mesocircuit hypothesis. Trends Neurosci 2010;33:1-9.

10. Whyte J, Myers R. Incidence of clinically significant responses to zolpidem among patients with disorders of consciousness: A preliminary placebo controlled trial. Am J Phys Med Rehabil 2009;88: 410-418.

\section{Disclosure}

M.N.B. Department of Physical Medicine and Rehabilitation, University of Michigan, 325 E. Eisenhower Pkwy, Ste 100, Ann Arbor, Ml 48108. Address correspondence to: M.N.B.; e-mail: mbomalas@med.umich.edu

Disclosure: nothing to disclose
S.R.S. Department of Physical Medicine and Rehabilitation, University of Michigan, Ann Arbor, Ml

Disclosure: nothing to disclose

Submitted for publication July 22, 2016; accepted December 29, 2016. 